\title{
Les entreprises sociales en quête de professionnalisation
} L'exercice difficile des associations d'aide à domicile aux personnes âgées

\section{Social enterprises in search of professionalism The challenge for elderly home-care associations}

\section{Cécile Clergeau et Annie Dussuet}

Numéro 294, novembre 2004

URI : https://id.erudit.org/iderudit/1022021ar

DOI : https://doi.org/10.7202/1022021ar

Aller au sommaire du numéro

Éditeur(s)

Institut de l'économie sociale (IES)

ISSN

1626-1682 (imprimé)

2261-2599 (numérique)

Découvrir la revue

Citer cet article

Clergeau, C. \& Dussuet, A. (2004). Les entreprises sociales en quête de professionnalisation : l'exercice difficile des associations d'aide à domicile aux personnes âgées. Revue internationale de l'économie sociale, (294), 39-53. https://doi.org/10.7202/1022021ar
Résumé de l'article

Le secteur de l'aide à domicile aux personnes âgées fait ressortir plusieurs modèles d'emploi, entre modèle " professionnel » et modèle " flexible ». Les auteurs s'appuient sur une enquête menée en 2002 auprès de vingt-quatre structures d'offre de service à domicile aux personnes âgées dans les départements du Maine-et-Loire et de la Loire-Atlantique. Elles montrent une étroite dépendance entre les modèles d'emploi et le type de service proposé par les organisations. Il apparaît ainsi que le type de service « aide à domicile ", inventé par l'économie sociale et solidaire, tente un compromis entre la logique professionnelle des services dits hospitaliers et la logique de la domesticité des services domestiques. Par là même, en structurant une relation de service dans le monde domestique, l'économie sociale et solidaire s'engage vers une professionnalisation de ces métiers. Après l'étude des processus entrepreneuriaux à l'oeuvre dans le domaine des services à domicile aux personnes âgées (cf. « Recma », n²93), ce nouvel article s’intéresse aux salariés prestataires du service. 


\section{LES ENTREPRISES SOCIALES EN QUÊTE DE PROFESSIONNALISATION} L'exercice difficile des associations d'aide à domicile aux personnes âgées

(*) Maître de conférences, LENCEBS université de Nantes, faculté des sciences économiques et de gestion (chemin de la Censive-duTertre, BP 52231, 44322 Nantes cedex 3).

Mél. Clergeau@sc-eco.univ-nantes.fr. $\left({ }^{* *}\right)$ Maître de conférences, UMR Gers-Iresco Paris, UFR de sociologie université de Nantes (chemin de la Censive-du-Tertre, BP 81227 44312 Nantes cedex 3). Mél. annie.dussuet@humana.univ-nantes.fr. par Cécile Clergeau (") et Annie Dussuet ${ }^{(*)}$

Le secteur de l'aide à domicile aux personnes âgées fait ressortir plusieurs modèles d'emploi, entre modèle "professionnel " et modèle "flexible". Les auteurs s'appuient sur une enquête menée en 2002 auprès de vingtquatre structures d'offre de service à domicile aux personnes âgées dans les départements du Maine-et-Loire et de la Loire-Atlantique. Elles montrent une étroite dépendance entre les modèles d'emploi et le type de service proposé par les organisations. Il apparaît ainsi que le type de service " aide à domicile ", inventé par l'économie sociale et solidaire, tente un compromis entre la logique professionnelle des services dits hospitaliers et la logique de la domesticité des services domestiques. Par là même, en structurant une relation de service dans le monde domestique, l'économie sociale et solidaire séngage vers une professionnalisation de ces métiers. Après l'étude des processus entrepreneuriaux à l'œuvre dans le domaine des services à domicile aux personnes âgées (cf. "Recma ", $n^{\circ} 293$ ), ce nouvel article sintéresse aux salariés prestataires du service.
(1) La Dares donne pour l'année 2000 un nombre moyen d'heures travaillées par salarié de neuf heures hebdomadaires dans les organismes prestataires, de dix heures dans les mandataires et de douze heures pour les organismes bi-actifs (Denantes, 2001). 'économie sociale et solidaire (ESS) occupe aujourd'hui une place de tout premier rang dans le secteur de l'aide à domicile aux personnes âgées. L'histoire de la prise en charge du vieillissement montre que la demande a été construite par les organismes de l'ESS en même temps qu'ils proposaient une offre de service. Les acteurs de l'ESS ont été de véritables entrepreneurs sociaux, transformant les représentations sociales et les comportements des personnes âgées et de leur entourage en même temps qu'ils structuraient leur intervention.

Les résultats de cette action de l'ESS sont pourtant ambigus en ce qui concerne les emplois développés. Occupés à $99 \%$ par des femmes, ils apparaissent marqués par une très grande précarité: prédominance de l'exercice à temps partiel avec des durées très faibles ${ }^{(1)}$, conjugué à une faible reconnaissance de qualification et des rémunérations très basses, souvent même proches des seuils de pauvreté... C'est ici un véritable défi lancé à ces organismes de l'ESS que de professionnaliser ces métiers de l'aide à domicile pour que son intervention dans ce secteur ne vienne pas renforcer le dualisme du marché du travail. 
Une enquête menée en 2002 sur les services à domicile aux personnes âgées auprès de vingt-quatre structures d'offre de service dans les deux départements du Maine-et-Loire et de la Loire-Atlantique (Clergeau, Dussuet, Nogues, Prouteau, Schieb-Bienfait, Urbain, 2002) nous conduit à considérer plusieurs modèles d'emploi dans ce secteur et nous permet de montrer une étroite dépendance entre les modèles d'emploi et le type de service proposé par les organisations. Nous montrons ainsi qu'entre un modèle d'emploi "professionnel " associé à un type de service " hospitalier " et un modèle "flexible » associé à un service " domestique », il existe un modèle de service inédit, inventé par les organisations de l'ESS, celui de l'" aide à domicile ", qui tente de professionnaliser une relation de service dans le monde domestique. Ce modèle intermédiaire de service est sans cesse menacé de dérives, soit vers le type hospitalier, soit, le plus souvent, vers le type domestique, entraînant alors l'emploi dans la précarité. Nous montrons alors que l'ESS joue un rôle fondamental dans le maintien d'un équilibre entre ces deux modèles, que l'on peut qualifier de " gouvernance médiatrice » entre l'ensemble des parties prenantes à la production de ce service. C'est cette gouvernance qui peut l'autoriser à engager une professionnalisation des activités d'aide aux personnes âgées.

\section{Entre le prescrit et le domestique, les services d'aide à domicile}

Les travaux sur l'économie des services en soulignent tous la dimension coproductive (Gadrey, 1994), même si l'implication du client-usager peut varier selon la nature du service (Zarifian, 2001). Les offreurs et les demandeurs co-construisent la demande et l'offre au sens où ils participent tous deux à la définition et à la mise en place du service rendu et où ils inscrivent dans l'espace public l'arrangement trouvé (Laville, Nyssens, 2001). Cette caractéristique co-productive du service le rend complexe, incertain et très dépendant de la qualité des interactions entre le client-usager et le prestataire (Bitner, Zeithaml, 2003). Et ceci est particulièrement crucial dans le domaine des services à la personne, tant la perception de la qualité par le client-usager est intrinsèquement liée à la perception du déroulement du processus de production, c'est-à-dire à la perception de la qualité des interactions co-productives (Grönroos, 1999).

Quatre éléments sont généralement mentionnés pour qualifier les services à la personne (Zarifian, 2001; Butté-Gérardin, 1999; Gomez, 1994; Gadrey, 1994):

- le degré de standardisation du service produit;

- le degré d'implication du client-usager dans la définition et la production du service;

- le degré de professionnalisation du prestataire;

- la nature des conventions de service qui en définissent la qualité.

C'est en nous appuyant sur ces travaux que nous identifions trois types de services d'aide aux personnes âgées. 
(2) Mais on observe aussi des effets de " contagion ": dans une structure qui offre plusieurs types de services et s'est structurée autour de SSIAD, le lancement d'un service de garde à domicile se fait sur ce même mode du service hospitalier.

\section{Les services hospitaliers}

Dans cette forme de service, on retrouve des caractéristiques proches de celles que l'on rencontre hors du domicile, dans les établissements hospitaliers de soins. Logiquement, les structures concernées sont plutôt celles qui délivrent des soins à domicile, qu'il s'agisse d'hospitalisation à domicile ou, plus couramment, de toilettes effectuées par des aides-soignantes dans des services de soins infirmiers à domicile ${ }^{(2)}$. Elles sont publiques, dépendant d'un hôpital, ou associatives.

Ces services sont définis par un prescripteur extérieur, souvent un médecin, c'est-à-dire un professionnel qualifié, qui définit un "protocole de soin » dont il délègue à un autre professionnel (infirmière, kinésithérapeute, aide-soignante...) tout ou partie de la réalisation. Si ce protocole de soin est établi sur mesure, il peut être décliné en une série d'actes codifiables objectivés par une nomenclature (d'actes médicaux, par exemple). Le service ne répond pas simplement à la demande de la personne âgée: le besoin est identifié à travers le diagnostic effectué par le prescripteur dont la légitimité dans ce domaine est établie par son pouvoir de déclencher le financement socialisé des prestations. La définition du service, tant de son contenu que de sa temporalité, est donc en partie " extérieure " à la relation entre prestataire et bénéficiaire.

Peut-être suffit-il, pour comprendre ce mode de définition du service, de se référer au vocabulaire employé par les acteurs, puisque ici on parle en " lits », comme dans les établissements hospitaliers. Le service délivré l'est donc à des bénéficiaires " occupant ces lits ", sans que soient prises en compte leurs caractéristiques personnelles. A la personne âgée n'est ainsi laissée aucune latitude de choix: les horaires sont imposés par les contraintes d'organisation de la structure, même si plusieurs interlocuteurs ont insisté sur leur bonne volonté pour respecter les souhaits horaires des personnes âgées. De même les personnes aidées n'ont-elles pas le choix de l'intervenant(e). La règle la plus généralement observée est celle d'une rotation systématique, les interventions étant le plus souvent quotidiennes, voire plus fréquentes.

Une dissociation s'opère alors entre le service et la personne qui le produit grâce à une définition objectivée. Dans ces conditions, les caractéristiques personnelles des intervenants apparaissent secondaires et il devient possible pour les organisations d'imposer des salariés dérogeant à l'image attendue par les bénéficiaires du service. On trouve donc plus souvent dans ce premier type de service des hommes, même s'ils sont minoritaires, ou des salariés d'origine étrangère.

Les services dits hospitaliers sont donc des services définis par des experts et rendus par des prestataires, tous professionnels. L'expert et le prestataire ont une compétence énoncée et reconnue par la puissance publique (diplôme) et par un syndicat professionnel. Cette labellisation constitue ici encore un gage de qualité, une certification, autorisant un a priori de confiance de la part de l'usager et contribuant de manière importante à la réduction de l'incertitude. 
(3) II s'agit souvent de structures proches d'associations intermédiaires.

\section{Les services domestiques}

A l'opposé de ce premier type de service, on peut distinguer un deuxième modèle que l'on nommera "domestique " parce qu'il emprunte nombre de traits à l'univers domestique. On peut y voir un exemple du monde domestique tel que défini par Boltanski et Thévenot (1991), mais aussi remarquer que le service proposé est défini plus ou moins explicitement en référence au travail domestique effectué gratuitement dans le cercle familial (Dussuet, 1997). On trouve dans ce cas de figure des organisations qui se sont donné comme objectif l'insertion par le travail de populations dites " éloignées de l'emploi " (3), ainsi que les associations mandataires, ou la partie mandataire des associations d'aide à domicile. On y trouve aussi quelques-unes des entreprises privées présentes sur le secteur.

Loin d'être l'objet d'une prescription d'un tiers, le service est défini ici comme ce qui répond à la demande du client, compris comme le bénéficiaire, avec ou sans son entourage familial. Dans les deux cas, l'expression des besoins et la définition de services correspondants ressortent essentiellement de la boîte noire familiale, du monde domestique. La question pour les structures adoptant ce deuxième modèle n'est pas celle de la légitimité à offrir tel ou tel service: la justification de l'offre est contenue dans le "besoin " du client. On a donc affaire à un service parfaitement "sur mesure ", qui peut même être "inventé " au gré de l'apparition de besoins comme le montre l'exemple d'une structure qui propose, en plus de services ménagers, un service d'entretien des tombes et du gardiennage d'animaux.

La plupart des structures qui offrent des services à domicile ne sont pas spécialisées. Dans de nombreux cas, l'employeur est la personne âgée bénéficiaire et l'association (dite mandataire) gère en son nom la sélection et le recrutement de l'agent prestataire, puis les formalités administratives. Ces structures ne sont pas à proprement parler offreuses d'un service à domicile, mais plutôt d'un service de mise en relation entre les deux partenaires de la co-production du service. Les organismes bi-actifs - à la fois prestataires et mandataires - opèrent le plus souvent une distinction très nette entre le fonctionnement de leurs deux types de service: en mandataire, les clients peuvent définir eux-mêmes la nature du service, les horaires et les caractéristiques du (ou de la) salarié(e) intervenant à domicile, alors qu'en prestataire, ces éléments leur sont imposés. En mandataire, les limites du service sont donc très floues et peu d'organismes ont un document les précisant. Le plus souvent, c'est aux salariées elles-mêmes que revient de décider au coup par coup ce qu'elles doivent (peuvent) faire dans le cadre de leur travail.

Le service est alors inséparable des personnes. Même si les tâches effectuées sont d'ordre matériel - ménage, nettoyage, repassage -, la relation entre salarié(e) prestataire et bénéficiaire du service est essentielle, parce que c’est à travers elle que se réalise la co-production du service. Les structures sont donc extrêmement attentives à la construction d'une relation de bonne qualité. Plutôt que de demander un service extérieur au cercle familial, 
les clients sont ici en position de " déléguer " la production de ce service à une tierce personne, l'intervenante à domicile. La relation marchande qui s'instaure reste néanmoins un supplétif du domaine domestique. Les conventions de service sont sous-tendues par un modèle familial et domestique.

\section{L'u aide à domicile ", un modèle de service inventé par l'économie sociale et solidaire}

Entre les services hospitaliers et les services domestiques, on peut caractériser un type intermédiaire auquel on réservera la désignation de « services d'aide à domicile ». On le rencontre dans des structures, le plus souvent associatives (mais ce sont aussi parfois des centres communaux d'action sociale, donc des structures publiques), qui ont une histoire longue dans le domaine de l'intervention à domicile, souvent à l'origine par l'emploi de travailleuses familiales, puis, quand elles se sont tournées vers le public des personnes âgées, avec les aides ménagères, aujourd'hui dénommées " aides à domicile».

On peut parler ici de co-définition du service dans la mesure où la réponse de ces structures est toujours initialisée par la visite à domicile d'un(e) responsable qui va, en coopération avec la personne âgée et éventuellement sa famille, s'attacher à recenser les besoins et à proposer des réponses, plus ou moins formalisées dans un protocole de service lui-même subdivisé en tâches précises. Le service est donc défini et négocié entre le client, sa famille et un tiers qui n'est pas le prestataire effectif et n'a pas l'autorité d'un prescripteur (il s'agit le plus souvent d'un[e] responsable de secteur). On est à mi-chemin entre la logique de la prescription propre aux services hospitaliers et la logique du choix propre aux services domestiques: la définition du service s'opère lors d'une véritable négociation où les principes de fonctionnement de la structure sont confrontés aux exigences des personnes aidées. Ce sont à la fois la nature des tâches, les horaires et la fréquence des interventions qui sont alors définis.

Le service d'aide à domicile vise à remplir une fonction générale d'" aide au maintien à domicile ». Contrairement à ce qui se passe dans les services hospitaliers, aucune nomenclature d'actes professionnels ne vient en soutenir la définition. Qu'elles soient associatives ou à but lucratif, privées ou publiques, employeuses ou mandataires, les organisations prestataires ont alors un rôle fondamental dans la définition de ce service. A l'extrême, lorsque ce rôle n'est pas assumé, le service ne peut être défini que par le client lui-même et rejoint alors le type domestique. Au contraire, lorsque la structure joue pleinement l'intermédiation, elle tend parfois à imposer ses principes et valeurs sur le mode d'un prescripteur et l'on retrouve la forme du service hospitalier. Mais, dans la plupart des cas, on se trouve dans une situation intermédiaire. Le client n'a alors qu'un choix relatif sur la question des horaires et des caractéristiques de l'agent prestataire. C'est sans doute dans les organismes bi-actifs, comportant à la fois un service prestataire et un service mandataire, que l'on perçoit le mieux les conséquences du positionnement en service domestique ou en service d'aide 
à domicile de la structure. En effet, lorsqu'elle est simplement mandataire, la structure, non employeuse, ne peut jouer pleinement l'intermédiation, le service glisse vers le type domestique. La forme juridique d'emploi détermine ainsi le mode de pilotage du service. En mandataire, il parait difficile pour les associations d'imposer une définition du service. Le client ne leur achète qu'un service administratif de gestion du contrat de travail. Le service à domicile est alors entièrement dominé par la logique domestique. En prestataire, les structures retrouvent une marge de manœuvre, en tant qu'employeuses, qui se traduit notamment dans leur préférence pour la rotation des intervenant $(\mathrm{e})$ s.

Celle-ci est vue comme souhaitable par les responsables, d'une part pour de simples raisons d'organisation (remplacements pendant les congés ou les formations), d'autre part parce qu' une trop grande personnalisation des relations est perçue comme néfaste. Toutefois, les usagers apparaissent encore plus réticents au changement de personnel que dans les services hospitaliers. Généralement, ils poussent à l'établissement de relations plus personnalisées avec "leur " aide habituelle, refusant même parfois les remplacements pendant les périodes d'absence ou de congé. Contrairement à ce que l'on pourrait penser, ce n'est pas quand le service intervient au plus près de l'intimité corporelle que l'attachement à un service personnalisé est le plus grand: il apparait plus facile de faire tourner les intervenant(e)s pour effectuer des toilettes que pour faire du ménage! Ce qui est en question est en fait la nature plus ou moins prescrite du service. La question de la rotation des intervenants peut ainsi être prise comme un indice du caractère "sur mesure " du service réalisé et donc (dans une certaine mesure) de son inscription dans le registre du domestique. Malgré les efforts des structures, cette personnalisation reste importante, imposant parfois une gestion du personnel peu conforme à un modèle professionnel, avec l'éviction de personnels mal acceptés en raison de leur origine ethnique, de leur âge... ou tout simplement de leur sexe.

Le service d'aide à domicile est ainsi un modèle de service qui tente un compromis entre la logique professionnelle de prescription du service hospitalier et celle de la domesticité. La sphère de l'aide à domicile réalise une situation intermédiaire où le pouvoir de définition du service n'appartient exclusivement ni à des experts ni à l'usager. Les organisations se positionnent ainsi dans un entre-deux entre les mondes hospitalier et domestique, mais sans modèles référentiels de conventions de service, ce qui les oblige à la création de normes, de systèmes d'emploi, de conventions touchant tant à la définition du service qu'à celle de sa qualité, tout en respectant cette exigence de référence au modèle de la solidarité auquel ils se substituent. Cette co-définition du service, mais aussi de ses conventions, est opérée par un processus de négociation qui requiert proximité et capacité de médiation. A cet égard, l'économie sociale et solidaire partage avec le monde domestique (Boltanski, Thévenot, 1991) des valeurs de civisme, de bienveillance, de confiance qui lui permettent très certainement de réaliser au mieux cette négociation et lui donnent en particulier 
une légitimité à s'arroger une fonction de prescription d'un protocole de service, mais aussi une fonction de définition d'un nouveau modèle d'emploi associé.

\section{Modèles de services, modèles d'emploi}

(4) Notons au passage qu'il s'agit bien de " ses " salariés: elle n'est pas simplement mandataire.
En caractérisant les types de services d'aide aux personnes âgées, ce sont aussi trois modèles d'organisation co-productive que nous étudions et donc trois modèles d'emploi. Les travaux de l'économie du travail (Gazier, 1993; Perrot, 1992) ont montré comment la nature des relations d'emploi (modalités contractuelles) était intrinsèquement liée à celle des relations de travail (la coordination, l'organisation et la standardisation des tâches, la participation aux décisions...), et ce en fonction de l'environnement économique des entreprises, mais aussi de l'organisation productive et de la gestion du capital humain. Les investigations menées auprès des structures proposant des services à domicile aux personnes âgées nous ont permis d'établir une même correspondance entre nature du service et de l'organisation productive et nature des relations de travail et d'emploi.

\section{L'emploi professionnel des services hospitaliers}

La légitimité du pouvoir de définition du service hospitalier est basée sur la professionnalité reconnue à l'expert par la puissance publique (diplôme) et par un syndicat professionnel; de même que la convention de qualité touchant à l'exécution des actes est fondée par la professionnalité du prestataire reconnue pareillement. Ces services impliquent donc un système d'emploi à caractère " professionnel ».

Ce type de services à domicile aux personnes âgées est ainsi le seul pour lequel sont exigés des diplômes lors des recrutements. On trouve en effet dans ces structures essentiellement des aides-soignantes, mais aussi quelques infirmières et, pour l'hospitalisation à domicile, des médecins. Les salarié(e)s de ces organisations sont aussi ceux (ou celles) qui sont les moins exposés à la précarité. Les contrats de travail, généralement à durée indéterminée et mensualisés, les lient directement à un seul employeur. La perspective de l'exercice à temps plein existe et les temps partiels sont généralement supérieurs au mi-temps.

La déclinaison du protocole de service en une série d'actes professionnels codifiés permet une organisation du travail proche de celle de l'hôpital. Le service n'est pas simplement produit par l'intervenante à domicile, il l'est par l'ensemble de la structure qui organise, en le répartissant, le travail de ses salariés ${ }^{(4)}$.

Comme à l'hôpital, on observe un système de tournées, composées d'une succession de visites aux différents domiciles. Le système de rotation permet en principe la conformité à la réglementation du travail (jours de congé, amplitude quotidienne, travail du week-end et des jours fériés) tout en assurant la continuité du service. 
Les déplacements d'un domicile à l'autre sont inclus dans le temps de travail. La journée de travail s'entend de l'arrivée au siège de l'organisation jusqu'au retour à ce même lieu après les différentes interventions. L'utilisation de véhicules de service appartenant à la structure définit comme situation de travail les temps de déplacement des salariées. D'une manière plus générale, lorsque, à défaut de voitures de service, les salarié(e)s utilisent leur véhicule personnel, un système de défraiement est mis en place, les déplacements intermédiaires restant inclus dans le service.

L'obligation d'un passage au siège de l'organisation structure le collectif de travail. Dans tous les cas étudiés, la tenue régulière, au minimum hebdomadaire, parfois quotidienne, de réunions de service permet la rencontre entre des salariées qui, dans l'exercice de leurs tâches, se trouvent totalement isolées à la fois de leurs collègues et de leur hiérarchie. Même si ces réunions ont essentiellement pour objet de coordonner les plannings, elles peuvent aussi fournir l'occasion d'une parole partagée sur les difficultés rencontrées individuellement auprès des personnes aidées, permettant la construction de normes professionnelles d'action.

\section{La précarité des services domestiques}

La définition du service domestique comme parfaitement ajusté aux besoins de la personne aidée implique une organisation du travail particulièrement flexible. Accepter les exigences horaires des clients, par exemple, signifie le plus souvent pour les salarié(e)s un éclatement des temps d'intervention et des chevauchements avec leurs propres rythmes sociaux. Pour les structures, cela signifie aussi la quasi-impossibilité d'établir des plannings cohérents. A l'extrême, cela veut dire embaucher du personnel toujours nouveau pour répondre à de nouvelles demandes. On trouve dans les structures adoptant ce modèle de service des temps de travail le plus souvent inférieurs au mi-temps. Le modèle de référence étant ici le gré à gré, on a une deuxième source de précarité: l'existence même de l'emploi est liée aux aléas biographiques de l'employeur. L'hospitalisation et a fortiori le décès se traduisent par un licenciement. Et même en écartant ces éventualités, l'employeur tend à ne recourir à la salariée qu'en fonction de ses besoins, ce qui oblige celle-ci à multiplier les contrats - et les employeurs - pour obtenir un revenu minimal et relativement régulier.

La définition du service comme réponse parfaitement ajustée au besoin du bénéficiaire implique aussi que le service se résume à la disponibilité: seuls les temps de présence des salariées au domicile des personnes aidées sont perçus comme temps de travail. Et encore, parfois pas entièrement, puisque la convention collective des " salariés de particulier employeur", applicable aux salariées en mandataire, prévoit la possibilité d'heures de "présence responsable ", censées être "sans travail effectif » et qui sont donc rémunérées

(5) Elles sont prévues dans le contrat de travail et peuvent représenter jusqu'aux trois quarts du temps de présence. avec un abattement de $30 \%{ }^{(5)}$. Cela exclut du décompte du temps de travail les déplacements des salariées entre les différents domiciles. De même, les moments de coordination et d'échange permettant de sortir du huis clos domestique ne leur sont guère accessibles, puisqu'ils ne peuvent être 
rémunérés en cas de système mandataire. La plupart des structures ayant adopté ce modèle ne proposent d'ailleurs de réunions qu'à titre volontaire et à un rythme au plus semestriel. Ainsi la construction d'un corpus de règles collectives permettant à une identité professionnelle d'émerger est-elle rendue difficile, sinon impossible.

Aucun diplôme n'est nécessaire pour exercer ce travail. Même s'il existe un diplôme d'aide à domicile, la méfiance signalée de nombreuses personnes âgées à l'égard des jeunes femmes diplômées montre que la compétence a ici une composante essentiellement privée. Elle est d'abord énoncée par le client qui définit ses attentes, puis confirmée ou infirmée au gré du temps et des relations spécifiques qui le lient à l'intervenante. Il ne s'agit donc pas d'une reconnaissance de qualification qui inscrirait le salarié dans une grille de classification (Lyon-Caen, 1988; Saglio, 1998) et lui permettrait d'accéder à une hiérarchie établie de rémunération.

La définition du service, laissée à la discrétion de l'utilisateur, ne permet pas une objectivation suffisante de celui-ci pour que soit reconnue la nécessité d'une qualification construite socialement, par le système scolaire par exemple. Seules des qualités personnelles sont requises, en apparence innées et qui ne semblent pouvoir être portées que par certaines personnes, des femmes, suffisamment âgées pour avoir dans leur propre vie privée rencontré l'expérience de l'aide aux personnes, que ce soit dans la maternité ou dans les relations filiales. Dans ces conditions, les structures ont du mal à recruter des salariés ne correspondant pas à ces représentations: hommes, jeunes, étrangères.

\section{Les services à domicile: une tentative de professionnalisation}

Ce système d'emploi se situe à mi-chemin entre les deux extrêmes présentés plus haut: emplois professionnels proches du salariat, d'une part, emplois flexibles et précaires de la domesticité, d'autre part. Mais on insistera ici sur la manière dont l'entre-deux que représente une co-définition du service peut être l'occasion pour les organisations de l'ESS de donner naissance à un type d'emploi original, à travers la professionnalisation.

On peut observer d'abord une forte réticence des structures concernées à embaucher n'importe quelle femme comme aide à domicile, au simple argument que celle-ci réalise chez elle les mêmes tâches domestiques. Les responsables affirment préférer des candidates porteuses de certification scolaire correspondant au service qu'elles seront amenées à réaliser: BEP sanitaire et social avec l'option «Maintien à domicile » dans l'idéal, ou au minimum un niveau $\mathrm{V}$ de formation, même si celle-ci concerne la vente ou la couture. Mais les associations se heurtent à un problème quasi insoluble lors des recrutements: le diplôme correspondant exactement à la profession d'aide à domicile, le certificat d'aptitude aux fonctions d'aide à domicile (Cafad), ne s'obtient pas en formation initiale, mais seulement en cours d'emploi ${ }^{(6)}$. Il y a donc très peu de demandeuses d'emploi qui en sont détentrices. Dès lors, les différentes structures ne se bornent pas à examiner les certifications scolaires des candidates, elles recrutent aussi sur

(6) La situation va évoluer avec le nouveau diplôme d'auxiliaire de vie sociale. 
(7) II s'agit ici simplement de l'application des règles de la convention collective de l'aide à domicile. d'autres critères, considérant en particulier que, dans ce domaine de l'aide à domicile, l'expérience acquise par les mères de famille ayant élevé leurs enfants est source de compétence. Certains responsables reconnaissent la difficulté de ces recrutements qui ne s'appuient pas sur une certification en soulignant le pari que représente l'embauche de ces salariées sans diplôme, mais tous sans exception s'accordent pour souligner la complexité du travail à effectuer par les aides à domicile et la nécessité de sélectionner les candidates sur leurs compétences. Du fait de l'absence d'exigence de diplôme pour exercer, on ne peut parler réellement de qualification (Dubar, 1996) pour ce type de service. Mais une reconnaissance socialisée des compétences de la salariée prestataire a lieu malgré tout, via la responsable de secteur, par l'organisme employeur, qui apparaît en position médiatrice.

Les associations ont par ailleurs une importante politique de formation en cours d'emploi, qui les conduit à encourager leurs salariées à préparer le Cafad ou bien encore à suivre des stages non diplômants. Cette politique apparaît désintéressée, puisque les salariées ont peu (Cafad) ou rien (formations non diplômantes) à gagner en termes de rémunération. Quant aux associations, elles ne peuvent espérer qu'un gain en termes de réputation, car les seuils légaux obligatoires sont généralement inférieurs à leurs investissements. Il faut donc souligner le caractère volontariste, pour les structures d'aide à domicile, de cette orientation vers la formation.

A travers cet accent mis sur les compétences et la nécessaire formation qu'elles exigent, on peut lire la volonté commune des associations de présenter le secteur comme réservé à un exercice professionnel. Mais elles se trouvent dans une position ambiguë, car, malgré leur volonté de faire prévaloir la nécessité de la professionnalisation, elles ne peuvent offrir à leurs salariées des conditions d'emploi qui manifestent un statut de professionnelles. On observe ainsi que, dans ces structures, l'organisation du travail se rapproche de celle des services domestiques: pas de tournées impliquant une division du travail entre des salariées relativement interchangeables, mais l'attribution à telle ou telle salariée de la prestation de service chez telle ou telle personne âgée, ce qui pourrait engager un glissement vers le modèle de la domesticité. Pourtant, le discours des responsables fait apparaittre une prise de distance vis-à-vis de l'établissement de relations interpersonnelles privilégiées: dans plusieurs associations sont énoncées des règles permettant d'éviter aussi bien l'assujettissement de la salariée aux besoins des personnes aidées que ses abus de pouvoir. D'autre part, s'il est rare que les structures fournissent un véhicule de service, elles prennent en compte, en général de façon forfaitaire, les temps et les frais de déplacement $^{(7)}$. Le temps de travail des salariées en est augmenté d'autant. Il inclut aussi, au moins dans les associations prestataires, des réunions de service au minimum mensuelles, auxquelles s'ajoutent des temps d'échange (groupes de parole) et de formation. Ces réunions, à visée technique, apparaissent fondamentales en ce qu'elles constituent, pour ces salariées dont la caractéristique est de travailler dans l'isolement de demeures privées, un lieu 
(8) Certaines peuvent être très importantes : une structure de notre échantillon dépasse les mille salariés. indispensable à l'émergence de règles collectives d'action, bases d'une possible professionnalité. Les responsables des structures concernées ont tous insisté sur l'importance de ces réunions en raison de la spécificité du travail des aides à domicile, mais ils disent aussi la difficulté à organiser ces rencontres qui ne sont pas considérées par les financeurs comme temps de travail, qui relèvent donc uniquement de leur propre décision et dont ils assument la responsabilité financière.

Les diverses réunions sont aussi le lieu de structuration d'un collectif de travail, dans lequel l'émergence d'une identité professionnelle peut déboucher sur des formes revendicatives. Les problèmes rencontrés par les salariées dans l'exercice de leur travail sortent alors du cadre interpersonnel pour devenir des revendications de reconnaissance professionnelle portant sur la définition de leur rôle. On constate ainsi, dans les plus grosses associations ${ }^{(8)}$, l'apparition de sections syndicales, ainsi que la mise en place des diverses institutions de représentation du personnel (CE, CHSCT...). Cette représentation du personnel tend à favoriser une évolution plus favorable des modalités d'emploi: définition de temps de travail au minimum à mi-temps, stabilisation des revenus par la mensualisation... Mais la démarche des associations d'aide à domicile comme employeuses est malaisée: d'un côté, les pressions de l'environnement les poussent à une flexibilité de type " domestique »; d'un autre côté, leur définition idéalisée du service d'aide aux personnes âgées les engage à "professionnaliser " leurs salariées, dans des conditions d'emploi qui s’y prêtent mal.

\section{La gouvernance médiatrice des organisations de l'économie sociale et solidaire}

Le modèle du " service d'aide à domicile " et les tentatives de professionnalisation des salariées qui lui sont associées nécessitent la réalisation de compromis entre des mondes qui s'opposent et sont sans cesse menacés par des dérives, soit vers la forme hospitalière, quand les structures imposent leur définition du service sans se préoccuper des formes de la demande, soit vers la forme domestique, quand elles tendent à s' effacer face aux exigences de personnalisation des clients, imposant alors à leurs salariées une forte précarité de l'emploi.

Les organisations de l'ESS apparaissent particulièrement bien placées pour réaliser les compromis nécessaires au maintien de cet équilibre. Une spécificité de l'activité du secteur est évidemment de pénétrer dans l'intimité des personnes aidées, un monde domestique dont de nombreux travaux ont montré la défiance à l'égard d'une intervention extérieure. Or il s'agit ici non seulement de rentrer dans ce cadre, mais d'y importer des règles qui permettent aux salariées d'effectuer leur tâche sans se soumettre à des relations de domesticité, d'affirmer dans le monde domestique une valeur à la professionnalité. Une seconde spécificité de cette activité est la place souvent primordiale de la famille de la personne âgée dans le processus de 
définition du service. Les associations le soulignent bien: ce sont parfois les enfants des personnes âgées qui demandent leur intervention. Et il est attendu par la famille que cette fonction d'aide au maintien à domicile, autrefois dévolue aux filles (aînées) et aujourd'hui externalisée, conserve certaines des valeurs familiales que sont la compassion et la bienveillance. Dans le même temps, les bénéficiaires comme leur famille comptent sur l'association pour les mettre en relation avec une personne " compétente ", soulevant la question d'une acception commune de la compétence. L'économie sociale est en quelque sorte chargée de gérer les tensions entre les nécessités d'une externalisation des activités domestiques de maintien à domicile vers des prestataires " compétents " et l'exigence d'un prolongement de la solidarité familiale.

Dans leurs travaux sur les économies de la grandeur, Boltanski et Thévenot (1991) offrent une analyse des principes supérieurs communs qui justifient les rapprochements possibles entre les individus, ce qui les amène à définir des « mondes » de représentations communes, de référentiels partagés soutenant la coordination des actions. Six mondes sont identifiés, dont le "monde domestique ", marqué par la référence à la tradition, à la figure du père ou d'un chef hiérarchique dont on estime le jugement. Il est empreint des valeurs de fidélité, de confiance, de bienveillance; l'habitude assure la stabilité des conduites individuelles et le devoir, plus que les droits, exige des conduites secourables à l'égard d'autrui et un rejet de l'égoïsme. Les logiques d'action mobilisées dans le monde domestique ne sont pas propres au seul monde familial ou privé. Ce monde " apparaît chaque fois que la recherche de ce qui est juste met l'accent sur les relations personnelles entre les agents » (p. 206). Par leur affirmation d'une prééminence accordée aux personnes, les associations et plus généralement les organisations de l'économie sociale offrent un compromis entre ces logiques d'action du monde domestique, mais aussi celles des autres mondes: monde de l'inspiration lorsque le projet est novateur, monde civique lorsque ce projet est d'intérêt général, monde marchand lorsqu'il sinscrit dans un marché concurrentiel et monde industriel lorsqu'il met en œuvre des règles ou des savoirfaire productifs plus ou moins codifiés.

Le monde domestique et le monde civique des associations partagent une même défiance à l'égard du monde marchand: l'idée selon laquelle l'argent peut pervertir les comportements. Boltanski et Thévenot soulignent l'idée communément admise dans le monde domestique selon laquelle les relations de confiance seraient perverties par l'intérêt et celle que l'on retrouve fréquemment dans les registres du monde civique selon laquelle le monde marchand serait celui de l'individualisme-roi. On peut probablement trouver dans cette défiance à l'égard du monde marchand un axe de rapprochement entre ces deux mondes expliquant la prédominance du secteur associatif dans l'aide aux personnes, d'une part, et celle subsidiaire des services publics locaux, d'autre part. Ces logiques d'actions domestiques et civiques, et les compromis qu'elles génèrent, sont autant de conventions qui vont gérer les relations entre le consommateur, sa famille et le prestataire. 
(9) L'introduction de l'allocation personnalisée à l'autonomie, qui suppose la définition de ce plan, pourrait contribuer à cette codification des services offerts, mais il faut noter que les structures prestataires seront alors dépossédées de leur pouvoir de prescription au profit de tiers extérieurs.
Elles permettent de créer la confiance dans la mesure où l'action du prestataire est motivée par des valeurs de solidarité, de bienveillance ou de compassion. La relation de service et la relation humaine qui la sous-tend ne sont pas engagées au nom de l'intérêt pécuniaire mais, par principe, au nom de la bienveillance et du bien commun, ce qui crée la confiance, réduit l'incertitude, sécurise l'usager et sa famille et peut être considéré comme gage de qualité et d'équité. Ces conventions régissent aussi les processus de pilotage et de contrôle de la prestation: elles apparaissent comme autant de repères, plus ou moins explicites, en permettant l'évaluation. C'est finalement l'ensemble du processus de co-production du service qui est facilité par une proximité née d'un partage de principes communs.

Les organisations de l'ESS peuvent ainsi se poser en médiatrices dans les rapports entre salariées prestataires du service et personnes aidées, garantes d'une position médiane entre les services hospitaliers, professionnels mais reposant sur une prescription d'actes codifiés à la définition desquels participent peu les bénéficiaires, et les services domestiques, personnalisés mais supposant un assujettissement des salariées. Pour maintenir cet équilibre, les structures mettent en œuvre divers moyens, repérés lors de l'enquête.

On peut distinguer d'abord ceux qui ont pour objet de contenir le glissement vers le type domestique. Pour ce faire, les organismes affirment leur propre définition du service à travers l'établissement de protocoles de service, établis sur mesure, mais qui vont ensuite être déclinés en une série de services génériques. Ce protocole de service permet de rompre le processus de personnalisation et éventuellement de protéger les partenaires contre les abus possibles: les salariés contre les exigences des personnes aidées, celles-ci contre les abus de pouvoir des intervenant(e)s. Nous avons ainsi pu observer l'établissement de règles ad hoc concernant les conditions de travail des salariées: tâches exclues du service parce que avilissantes ou trop pénibles étant donné les conditions de réalisation (saleté, manque de matériel), mais aussi règles de rotation destinées à éviter un attachement trop important, une responsabilisation trop forte. Les organismes employeurs interviennent aussi pour dénouer des conflits nés de malentendus ou de soupçons concernant l'honnêteté des intervenantes. Cette médiation demande du doigté pour ne pas entraîner systématiquement la rupture. Ce protocole de service se développe d'autant plus dans l'activité des associations qu'elles interviennent auprès de malades, de personnes très dépendantes ou de personnes présentant un handicap: on doit alors définir un véritable "plan d'aide " ${ }^{(9)}$. Le risque de dérives concerne alors la forme hospitalière. Là encore, on peut relever des tentatives pour contrer cette dérive par l'attention aux " personnes » et à leurs spécificités. On notera en particulier les efforts des organisations pour reconnaittre l'entourage de la personne âgée comme un système global de production domestique familiale, faire une place aux aidants familiaux. Plusieurs associations développent ainsi des actions de formation en direction des « aidants familiaux » destinées à créer, autour de la personne aidée, un ensemble mixte 
de personnes professionnelles et profanes, salariées et bénévoles, concourant à la production du service de maintien à domicile.

Toutefois, l'enquête montre comment cette activité médiatrice des associations a été handicapée dans les années récentes par les contraintes provenant de l'environnement institutionnel. La création de structures mandataires, en particulier, constitue un risque de dérive vers la forme domestique: placées en situation de concurrence, certaines organisations ont quelque peu délaissé l'objectif de professionnalisation de leurs salariées pour privilégier un impératif de pérennisation de l'activité, au prix de la précarisation des emplois. Il faut remarquer aussi que, dans les organismes bi-actifs, qui ont à la fois une activité prestataire et une activité mandataire, le système d'emploi de l'aide à domicile semble mieux résister que dans ceux qui n'ont qu'une activité mandataire. De même, dans les organismes qui interviennent dans des activités variées (soins infirmiers, garde à domicile, aide à domicile) et qui emploient pour l'une d'entre elles des professionnelles (infirmières, aides-soignantes ou travailleuses familiales), la professionnalisation des salariées de l'aide à domicile apparaît plus facile, le système d'emploi professionnel tendant généralement à l'emporter pour l'ensemble de la structure.

S'il n'existe pas à proprement parler, dans le secteur des services à domicile aux personnes âgées, de système d'emploi spécifique à l'ESS, des structures publiques ou privées lucratives pouvant présenter les mêmes caractéristiques, la question de la professionnalisation des salariées du secteur est porteuse d'enjeux majeurs, puisqu'elle touche à la définition même du service délivré. Et l'ESS se voit ici conférer un rôle majeur, parce qu'elle est aujourd'hui l'employeur le plus important dans ce secteur, mais aussi parce que son histoire et ses valeurs lui donnent un rôle de gouvernance médiatrice favorisant l'émergence de nouvelles conventions de service et d'une professionnalisation des acteurs. Les difficiles compromis qu'elle tente de faire émerger exigent d'être pris en compte par les politiques de l'emploi, en quête de "gisements d'emplois nouveaux ", qui peuvent ici apparaître intempestives. 


\section{Bibliographie}

Bitner M. J., Zeithaml V. A. (2003), Services marketing, integrating customer focus across the firm, MacGraw-Hill.

Boltanski L., Thévenot L. (1991), De la justification: les économies de la grandeur, Paris, Gallimard.

Butté-Gérardin I. (1999), L'économie des services de proximité aux personnes, le cas du soutien à domicile aux personnes âgées, L'Harmattan. Clergeau C., Dussuet A., Nogues H., Prouteau L., Schieb-Bienfait N., Urbain C. (2002), Léconomie sociale et solidaire et les services à domicile aux personnes âgées, rapport à la préfecture de la région Pays de la Loire, $277 \mathrm{p}$.

Denantes G. (2001), « Les emplois familiaux et les organismes de services aux personnes en 2000 ", Premières informations et premières synthèses 39.1.

Dubar C. (1996), « La sociologie du travail face à la qualification et à la compétence ", Sociologie du travail, $\mathrm{n}^{\circ} 2$.

Dussuet A. (1997), Logiques domestiques: essai sur les représentations du travail domestique chez les femmes actives de milieu populaire, Paris, L'Harmattan.
Gadrey J. (1994), « Les relations de service dans le secteur marchand ", in J. De Bandt et J. Gadrey (sous la dir. de), Relations de services et marchés de services, Paris, CNRS Editions.

Gazier B. (1993), Les stratégies des ressources humaines, Paris, La Découverte.

Gomez P. Y. (1994), Qualité et théorie des conventions, Paris, Economica.

Grönroos C. (1999), « Le marketing des services: consommation et marketing de processus ", Revue française de marketing, $\mathrm{n}^{\circ} 171$.

Laville J.-L., Nyssens M. (2001), Les services sociaux entre associations, Etat et marché, l'aide à domicile aux personnes âgées, La Découverte, Paris.

Lyon-Caen A. (1988), « Les systèmes de classifications professionnelles ", Travail et Emploi, $n^{\circ} 38$.

Perrot A. (1992), Les nouvelles théories $d u$ marché du travail, La Découverte, Paris.

Saglio J. (1998), " Qualifications et classifications ", Le monde du travail, J. Kergoat, J. Boutet, Jacot, D. Linhart, Paris, La Découverte. Zarifian P. (2001), " Valeur de service et compétence ", La relation de services, regards croisés, L'Harmattan, "Cahiers du genre ", nº 28. 